Research Article

\title{
Comparative Regression Analysis for Estimating Resonant Frequency of C-Like Patch Antennas
}

\author{
Umut Özkaya $\mathbb{D}^{1},{ }^{1}$ Enes Yiğit $\left(\mathbb{D},{ }^{2}\right.$ Levent Seyfi $\mathbb{D}^{1},{ }^{1}$ Şaban Öztürk $\left(\mathbb{D},{ }^{3}\right.$ and Dilbag Singh ${ }^{4}{ }^{4}$ \\ ${ }^{1}$ Department of Electrical and Electronics Engineering, Konya Technical University, Konya, Turkey \\ ${ }^{2}$ Department of Electrical and Electronics Engineering, Karamanoğlu Mehmetbey University, Karaman, Turkey \\ ${ }^{3}$ Department of Electrical and Electronics Engineering, Amasya University, Amasya, Turkey \\ ${ }^{4}$ Computer Science Engineering, School of Engineering and Applied Sciences, Bennett University, Greater Noida 201310, India \\ Correspondence should be addressed to Umut Özkaya; uozkaya@ktun.edu.tr
}

Received 13 May 2020; Revised 31 July 2020; Accepted 30 September 2020; Published 8 February 2021

Academic Editor: Thomas Hanne

Copyright (c) 2021 Umut Özkaya et al. This is an open access article distributed under the Creative Commons Attribution License, which permits unrestricted use, distribution, and reproduction in any medium, provided the original work is properly cited.

\begin{abstract}
This study provides a comparative analysis of regression techniques to estimate the operating frequency of the C-like microstrip antenna. The performance of well-known regression techniques such as linear regression (LR), regression tree (RT), support vector regression (SVR), Gaussian regression (GR), and artificial neural network (ANN) is tested. For this purpose, 160 C-like microstrip antennas are simulated, of which 145 are used for training of regression techniques and 15 for testing. From the evaluated results, it is found that the pure quadratic Gaussian regression (PQGR) technique has the lowest error rates with 0.0109 mean absolute error (MAE), 0.0087 median error (ME), 0.0002 mean squared error (MSE), 0.0156 root mean squared error (RMSE), and 0.5981 average percentage error (APE). As can be seen in the comparative analysis, the PQGR method outperforms other regression methods on simulation and measurement data. Experimental analysis shows that the resonant frequency of the C-like patch antennas can be calculated very close to measurements.
\end{abstract}

\section{Introduction}

Microstrip patch antennas constitute a wide field of research in the literature due to their advantages such as being light, small, easy in production, low cost, and easy integration into a system. Thanks to these advantages, it has been used with different designs in fields such as wireless communication systems, remote sensing systems, and medical and radar applications [1]. The design of microstrip patch antennas is achieved by coating the conductors on the upper and lower surfaces of thin dielectric material. One surface acts as a ground, while the other emits electromagnetic radiation. Microstrip patch antennas have disadvantages such as low gain of receiver and narrow bandwidth. In the literature, patch antennas with square, rectangular, triangular, and circle geometry are so common and easy to analyze theoretically [2-4]. In these types of antennas, different gains can be obtained by using different patch geometry and feeding techniques. Changes in antenna geometry can increase the effective electric field. Patch sizes and ground plane geometry can affect resonant frequency. Besides, the feed point location and feeding technique have high effects on the resonant frequency. The resonant frequency of the antenna is inversely proportional to the patch dimensions. In the literature, there can be found various shaped microstrip antennas such as C, E, H, and L shapes [5-9]. Such antennas are generally symmetrically loaded concerning the edge of the patch, while the slot antennas consist of asymmetric notches on one side of the patch. While symmetric antennas are easy to analyze, asymmetric slot antennas cannot be expressed in basic mathematical equations [10-18]. Therefore, analysis of the C-like slot antenna is performed by choosing different feed types, feed point location, and dielectric materials in this study.

One of the most important parameters for the microstrip patch antenna is the resonant frequency. The 
fact that antenna has different structure geometry rather than traditional geometry is a factor that makes the analysis difficult. On the other hand, microstrip antennas can be accurately simulated via software technology. The developed method of moments (MoM) uses the Maxwell equations and performs the necessary analyses. Since the cost of purchasing these methods in the software packages is very high, the designers tried to overcome this problem with artificial intelligence methods. Thanks to the intuitive and supervised methods that artificial intelligence offers to the users, they were able to approach the solution of the problems faster and more efficiently. These methods include artificial neural networks (ANNs) [19], regression analysis methods (RAMs) [20], support vector regression (SVR) [21], and regression trees (RT) [22]. In the literature, optimization algorithms and ANN are often preferred to estimate the resonant frequency of microstrip patch antennas. SVR [23], adaptive neurofuzzy interaction system (ANFIS) [24], and artificial bee colony (ABC) optimization are also widely used.

In this study, a detailed comparative analysis is performed to estimate the operating frequency of the C-like microstrip patch antenna. In this context, linear and Gaussian regression analyses, SVR, RT, and ANN are used. The pure quadratic Gaussian regression (PQGR) technique has achieved the highest performance. In PQGR analysis, dielectric material height $(h)$, C-like microstrip patch antenna dimensions $(L, W, 1, w, d)$, and relative dielectric constant $\left(\varepsilon_{r}\right)$ are given as input, and operating frequency $\left(f_{t}\right)$ is estimated as output. $160 \mathrm{C}$-like microstrip antennas are simulated in computer-based software combined with computational electromagnetic (CEM) software [25] for training and testing. While the simulated 145 antennas were used for training, the remaining 15 antennas are used for the test process. For comparison with the literature, the test antennas are selected as in [26]. To test the performance of the PQGR technique, a 6-fold cross-validation technique is applied. As a result of PQGR analysis, resonant frequency is converged with the best values of $0.0109 \mathrm{MAE}, 0.0087 \mathrm{ME}$, 0.0002 MSE, 0.0156 RMSE, and 0.5981 APE. The main contributions of the proposed PQGR method are as follows:

(i) C-shaped microstrip antenna data are analyzed with regression methods

(ii) Proposed PQGR model has higher performance than other regression methods

(iii) As seen in comparative results, the proposed PQGR models have low error metrics

(iv) In the testing phase, simulation and real data were evaluated in the proposed method

The outline of this study is as follows. In the next section, the design parameters of the antennas and the regression analysis methods are presented. In Section 3, comparative analyses are presented. In the last section, the study is summarized and future directions are mentioned.

\section{Material and Methods}

2.1. Material. As seen in Figure 1, C-like microstrip antenna's length and width are indicated as $L$ and $W$. The thickness of dielectric material is represented by $h$, and also its relative permeability is $\varepsilon_{r}$. In the $x-y$ coordinate system, the coaxial feed point is defined as $\left(x_{0}, y_{0}\right)$. There is an $l \times w$ slot in the rectangular patch. $d$ indicates the upper distance of the slot. The designed C-like antenna data are simulated with seven variables and form the input of the regression analysis. The resonant frequency is available as output.

The microstrip patch antennas are designed for use in ultra-high frequency (UHF) band applications. It should have a resonant frequency of 1.15-3.335 GHz. 160 different antennas were simulated with CEM software HyperLynx ${ }^{\circledR}$ 3D EM [27]. To provide a homogeneous data distribution, five different group values are created in the antenna dimensions. As given in Table 1, each group has 32 antenna data and outer dimensions of groups were 30,$20 ; 35,25 ; 40$, 30; 45, 35; and 50, 40 including different parameters of $l, w$, $d, h$, and relative permittivity $\varepsilon_{r}$. In order to compare with the literature [26], the coaxial feed point was determined as $x_{0}=5 \mathrm{~mm}$ and $y_{0}=5 \mathrm{~mm}$. 1-volt wave source was used for power supply. A total of 160 different antenna parameters were generated between 1 and $5 \mathrm{GHz}$ in CEM simulation.

\subsection{Techniques}

2.2.1. Linear Regression. Dependent variables can be expressed by many independent variables. Multiple linear regression analysis is the method used to explain the relationship between two and more independent variables affecting a variable in a linear model and to determine the effects of these independent variables. Multiple linear regression equation:

$$
Y=a+b_{1} X_{1}+b_{2} X_{2}+\ldots b_{m} X_{m}+\varepsilon
$$

where $Y$ is a dependent variable, $X_{1}, X_{2}, \ldots, X_{m}$ are independent variables, $b_{1}, b_{2}, b_{3}, \ldots, b_{m}$ are regression coefficients, and finally $\varepsilon$ represents the error term. $a$ is the regression constant. $b$ values are also called partial regression or partial slope coefficients. In multiple linear regression, there is no correlation between the number of independent variables and predictive accuracy [28]. Therefore, it is vital to determine the number of independent variables in terms of the reliability of estimation. In this study, linear, interaction linear, and stepwise linear regressions were used.

2.2.2. Regression Tree. Regression trees determine output value by integrating the regression method at the end of the model unlike the classification problem. The prediction results of regression trees are lower than those of other regression techniques. Regression trees can perform an efficient calculation by creating segmented linear models. First, a fixed tree model is created and the linear regression analysis is applied to the data in each node [29]. Residuals are calculated by adapting the nodes to the regression model. Since the complexity of the model is shared between the tree 


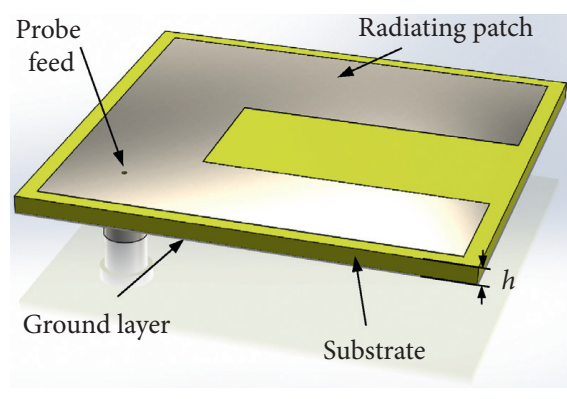

(a)

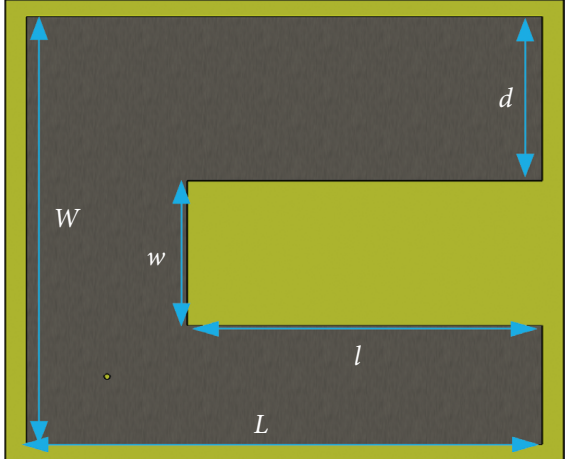

(b)

Figure 1: C-like microstrip patch antenna geometry. (a) Isometric view. (b) Front view.

TABLE 1: Simulated slot antenna parameters.

\begin{tabular}{|c|c|c|c|c|c|c|}
\hline \multicolumn{5}{|c|}{ Patch dimensions (mm) } & \multirow[b]{2}{*}{$h(\mathrm{~mm})$} & \multirow[b]{2}{*}{$\varepsilon_{r}$} \\
\hline $\mathbf{L}$ & $W$ & $l$ & $w$ & $d$ & & \\
\hline 30 & 20 & $10 ; 20$ & $5 ; 10$ & $3 ; 6$ & & \\
\hline 35 & 25 & $15 ; 25$ & $7 ; 12$ & $8 ; 10$ & & \\
\hline 40 & 30 & $15 ; 30$ & $7.5 ; 15$ & $5 ; 10$ & $1.6 ; 2.5$ & $2.33 ; 4.4$ \\
\hline 45 & 35 & $25 ; 30$ & $5 ; 10$ & $20 ; 25$ & & \\
\hline 50 & 40 & $20 ; 40$ & $10 ; 20$ & $7 ; 14$ & & \\
\hline
\end{tabular}

structure and the nodes, the complexity of the tree structure is reduced. For this reason, the complexity of the tree structure should be taken into consideration before the design of the model. As the complexity of the model increases, it requires a lot of data for training. Therefore, the size of the tree and the number of nodes should be determined according to the number of data. In this study, fine, medium, and coarse regression tree methods were used.

2.2.3. Support Vector Regression. In regression analysis, SVR is the most widely used type of support vector machine (SVM). The basic idea in SVM includes the determination of the regression function [30]. There are some differences in SVR from standard SVM operations. If the training data are defined as $\left\{\left(x_{1}, y_{1}\right),\left(x_{2}, y_{2}\right), \ldots,\left(x_{l}, y_{l}\right)\right\}$, some deviations may occur in objective function $f(x)$. To define the linear objective function,

$$
f(x)=(w, x)+b .
$$

The solution of the optimization problem in equation (3) can be approached by reducing Euclidean norm $\|w\|_{2}$.

$$
\text { Minimize } \frac{1}{2}\|\omega\|^{2} \text { subject to }\left\{\begin{array}{l}
y_{i}-\left(\omega, x_{i}\right)-b \leq \varepsilon \\
\left(\omega, x_{i}\right)+b-y_{i} \leq \varepsilon
\end{array}\right\} \text {. }
$$

For the objective function in equation (3), it is possible to converge at all values $\left(x_{i}, y_{i}\right)$ with a certain sensitivity $(\varepsilon)$. To deal with some constraints of the optimization problem, the objective function needs to be modified with some slack variables.

$$
\begin{aligned}
\operatorname{Minimize} & \frac{1}{2}\|\omega\|^{2}+\mathrm{C} \sum_{i=1}^{l}\left(\xi_{i}+\xi_{i}^{*}\right) \\
\text { subject to } & \left\{\begin{array}{c}
y_{i}-\left(\omega, x_{i}\right)-b \leq \varepsilon+\xi_{i} \\
\left(\omega, x_{i}\right)+b-y_{i} \leq \varepsilon+\xi_{i}^{*} \\
\xi_{i}, \xi_{i}^{*} \geq 0
\end{array}\right\} .
\end{aligned}
$$

In this study, linear, quadratic, cubic, fine, medium, and coarse SVR methods were used to analyze these antenna data.

2.2.4. Gaussian Regression. In the Gaussian process, finite subsets are created with multivariate Gaussian distribution with many variables. $N$ number of observations is $y=\left\{y_{1}, \ldots\right.$, $\left.y_{N}\right\}$, which can be defined as Gaussian distribution. In general, the mean Gaussian operation is assumed to be 0 in each observation. Covariance function is necessary to establish a relationship between observations [31]. The covariance function for the quadratic exponential function is defined as follows:

$$
k\left(x, x^{1}\right)=\sigma_{f}^{2} \exp \left[\frac{-\left(x-x^{1}\right)^{2}}{2 l^{2}}\right]
$$

The maximum covariance value that can be obtained as $\sigma_{f}^{2}$. When $x$ approaches $x^{l}$, covariance gets the maximum value. This means that there is a close relationship between $f(x)$ and $f$ $\left(x^{l}\right)$. The covariance value decreases if $x$ value moves away from 
$x^{2}$. In this study, rational quadratic, squared exponential, Matern $5 / 2$, and exponential Gaussian regression methods were preferred to analyze these antenna data.

2.2.5. Artificial Neural Network. Artificial neural network (ANN) is one of the artificial intelligence techniques. It was developed by imitating the stimulation and information received from organs to the brain via neurons. In these days, it is common to be used in almost all computational sciences. ANNs can also be thought of as a black box that processes input and generates outputs. This system processes information in parallel and learns the principle that connection coefficients between neurons are updated by minimizing the error.

An ANN model includes input, hidden, and output layers. Each layer has neurons that are connected utilizing their weight coefficients. The input and output layer can contain many neurons that are equal to the number of input and output. However, the number of neurons in the hidden layer depends entirely on the input. $(n+1) / 2$ or $2 n+1$ neurons can be used in the hidden layer, where $n$ is the number of inputs. A different number of neurons may be used depending on the nature of the problem. The less number of neurons will reduce the learning ability of the system [23].

$$
\begin{aligned}
& Y_{i}=f\left(\sum_{i=1}^{n} X_{h} V_{i h}\right), \\
& Z_{j}=f\left(\sum_{i=1}^{p} Y_{i} V_{j i}\right),
\end{aligned}
$$

where inputs are defined as $X$, the output of hidden layers is $Y$, and $Z$ is output. $V$ and $W$, respectively, indicate weights between the input-hidden layer and hidden-output layer in equations (6) and (7) $[32,33]$.

Learnable parameters in ANN architecture are updated either in the feed-forward or backpropagation phases. In the feed-forward, existing weight coefficients are obtained from neurons and ultimately the outputs of the system. The total error is calculated between predictions and the actual values. The error value is propagated as backward and then weight coefficients in the connections are updated. This process is iterated either for a specific number or until a given error threshold is reached.

2.2.6. Metrics. Some metrics are needed to evaluate the obtained results. Four metrics were used in this study. These are MAE in equation (8), ME in equation (9), MSE in equation (10), RMSE in equation (11), and APE in equation (12):

$$
\begin{aligned}
\mathrm{MAE} & =\operatorname{mean}_{i=1, n}|e|_{i}, \\
\mathrm{ME} & =\operatorname{median}_{i=1, n}\left|e_{i}\right|, \\
\mathrm{MSE} & =\operatorname{median}_{i=1, n}\left|e_{i}^{2}\right|,
\end{aligned}
$$

$$
\begin{aligned}
\text { RMSE } & =\sqrt{\operatorname{median}_{i=1, n}\left|e_{i}^{2}\right|}, \\
\mathrm{APE} & =\frac{\sum_{i=1}^{N}\left|f_{\mathrm{SIMi}}-f_{\mathrm{COMi}}\right|}{N} .
\end{aligned}
$$

2.2.7. Training and Validation. 160 CEM data were divided into 145 and 15 as training and validation, respectively. All training operations were performed on Intel Core i7-7700 HQ 2.8 GHz processor with $16 \mathrm{~GB}$ RAM in Matlab software. The metrics in this study were evaluated for accuracy of obtained resonant frequencies by proposed methods. Figure 2 shows how to generate antenna data from the CEM program.

The K-fold cross-validation method was used to prove the validity of the proposed methods. The cross-validation process divides the existing data set into equal subsets for training and validation processes. This process is divided into $\mathrm{K}$ subsets and repeated $\mathrm{K}$ times. Each subset must be used only once for validation. In this way, all data are used for both training and validation. Figure 3 illustrates the 6fold cross-validation process. All data (\# 160) were divided into 6 subsets $(5 \times \# 29$ and \# 15). For each iteration, 145 CEM data (CEMs) were used for training and 15 CEMs were used for validation.

2.2.8. Proposed Method. Some statistical metrics were used between simulation and calculated resonant frequency values to compare the performance of proposed regression analysis methods. These metrics are mean absolute error (MAE), median error (ME), mean squared error (RMSE), and root mean squared error (RMSE). In Figure 4, the proposed method is tried to be schematized. $f_{r S I M}$ and $f_{r C O M}$ represent simulated and computed resonant frequencies, respectively. In this study, the dataset was divided into six subsample sets within the scope of 6 cross-validations. Subsample 6 was reserved for testing. The proposed methods in the comparative analysis are LR, interaction LR, stepwise LR, fine RT, medium RT, coarse RT, linear SVR, quadratic SVR, cubic SVR, fine Gaussian SVR, medium Gaussian SVR, coarse Gaussian SVR, rational quadratic GR, squared exponential GR, Matern 5/2 GR, exponential GR, PQGR, and ANN.

\section{Results and Discussion}

The obtained results by the proposed method are given in Table 2. Primarily, subsample 6 without measurement data was used to test algorithms. It includes 15 CEMs. When metric performances were evaluated, the highest error in MAE belonged to coarse TR with 0.3521 . The lowest error in MAE was obtained with 0.0109 in PQGR. Considering ME, LR showed the worst performance with a value of 0.2993 . The lowest value of $0.0087 \mathrm{ME}$ was obtained with PQGR. In the context of MSE, the LR method had the worst performance with 0.1274 error value. PQGR was the most successful one with MSE value of 0.0002. As with other error 


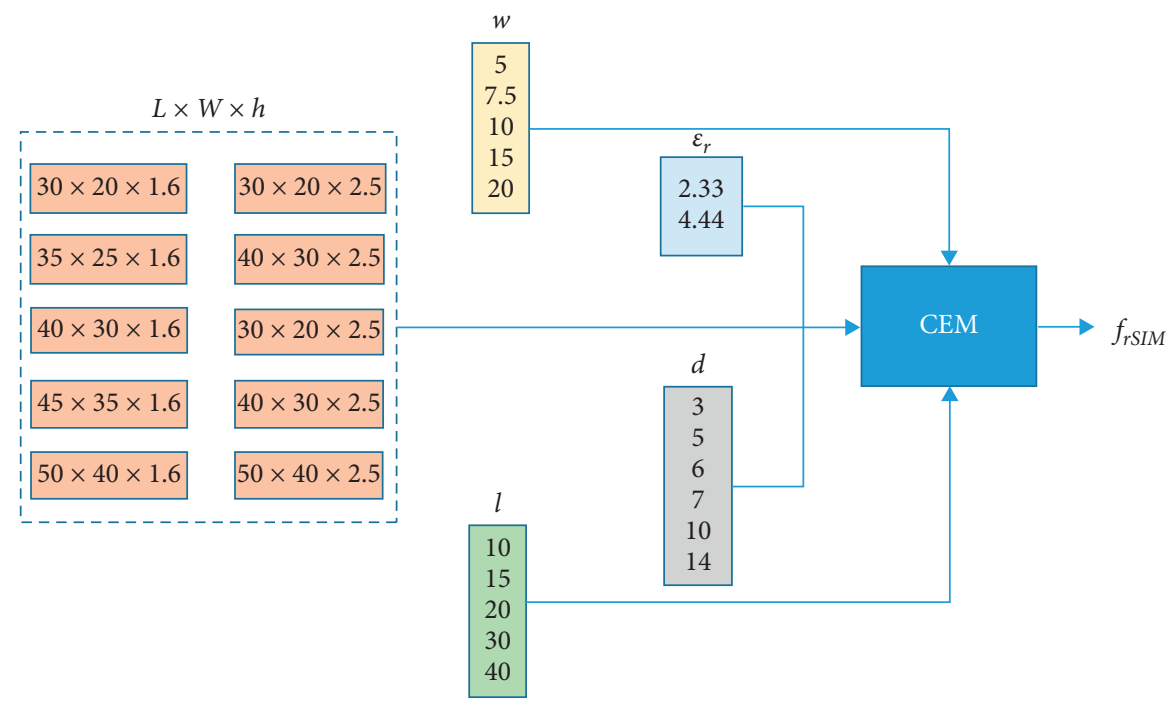

Figure 2: Data generation in CEM.

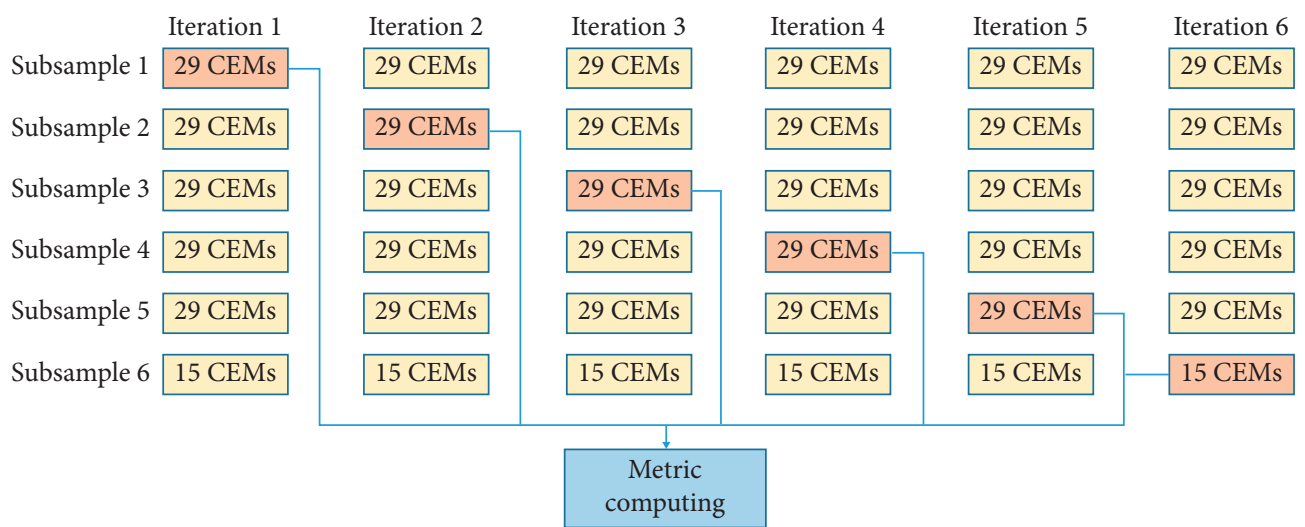

Validation

Traning

FIgURE 3: 6-fold cross-validation process.

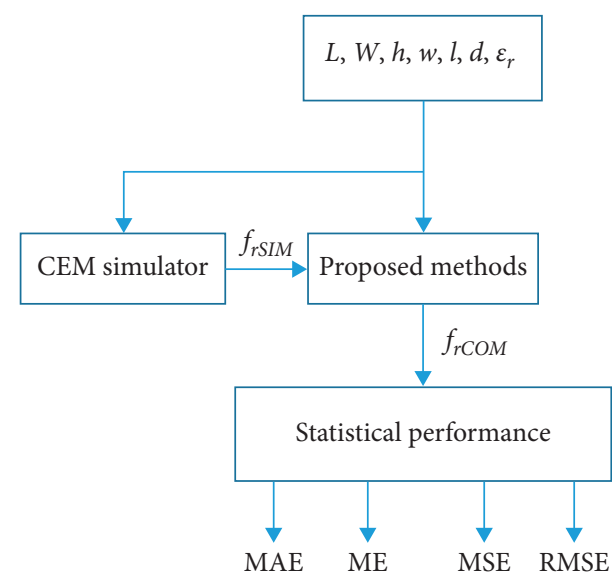

Figure 4: Proposed method. metrics, LR had the lowest performance with RMSE of 0.3569. PQGR was the best convergence of resonant frequency with 0.0156 RMSE value.

The PQGR method was evaluated within the scope of 6fold cross-validation as shown in Table 3. The dataset was divided into an equal number of subsamples 1-6. The highest error values were obtained as 0.0537 MAE, 0.0239 ME, 0.0044 MSE, and 0.0662 RMSE. The highest performance is $0.0109 \mathrm{MAE}, 0.0087 \mathrm{ME}, 0.0002 \mathrm{MSE}$, and 0.0156 RMSE for the subsample 6 dataset. The mean error values for all subsamples were 0.0292 MAE, 0.0149 ME, 0.0011 MSE, and 0.0278 RMSE. Scatter diagrams about test data are shown in Figure 5.

The trained PQGR model was tested in the subsample with six datasets. This dataset was also evaluated in the concept of MAE, ME, MSE, and RMSE. In this way, the performance of the PQGR model can be verified and 
TABLE 2: Metric performance of proposed methods.

\begin{tabular}{lcccc}
\hline Proposed methods & MAE & ME & MSE & 0.1274 \\
LR & 0.3246 & 0.2993 & 0.1237 & 0.0371 \\
Interaction LR & 0.1960 & 0.1768 & 0.0050 & 0.3517 \\
Stepwise LR & 0.1708 & 0.1680 & 0.0361 & 0.1925 \\
Fine TR & 0.0729 & 0.0457 & 0.0494 & 0.0705 \\
Medium TR & 0.1935 & 0.1633 & 0.0446 & 0.0122 \\
Coarse TR & 0.3521 & 0.2807 & 0.0289 & 0.2223 \\
Linear SVR & 0.3181 & 0.2588 & 0.0038 & 0.1105 \\
Quadratic SVR & 0.1533 & 0.0866 & 0.0207 & 0.1700 \\
Cubic SVR & 0.1455 & 0.1374 & 0.0647 & 0.0619 \\
Fine Gaussian SVR & 0.3371 & 0.2854 & 0.0014 & 0.1437 \\
Medium Gaussian SVR & 0.1896 & 0.1478 & 0.2545 \\
Coarse Gaussian SVR & 0.2990 & 0.2545 & 0.0050 \\
Rational quadratic GR & 0.0801 & 0.0669 & 0.0279 \\
Squared exponential GR & 0.0801 & 0.0669 & 0.0368 \\
Matern 5/2 GR & 0.0828 & 0.0709 & 0.0368 \\
Exponential GR & 0.1250 & 0.1341 & 0.0709 \\
PQGR & 0.0109 & 0.0087 & 0.1670 \\
ANN & 0.3228 & 0.2369 & 0.0156 \\
\hline
\end{tabular}

TABLE 3: 6-fold cross-validation for PQGR.

\begin{tabular}{lcccc}
\hline Sample types & MAE & ME & MSE & RMSE \\
\hline Subsample 1 & 0.0117 & 0.0094 & 0.0003 & 0.0006 \\
Subsample 2 & 0.0537 & 0.0239 & 0.0044 & 0.0240 \\
Subsample 3 & 0.0183 & 0.0128 & 0.0003 & 0.0662 \\
Subsample 4 & 0.0429 & 0.0192 & 0.0006 & 0.0187 \\
Subsample 5 & 0.0375 & 0.0152 & 0.0002 & 0.0253 \\
Subsample 6 & 0.0109 & 0.0087 & 0.0011 & 0.0156 \\
Average & 0.0292 & 0.0149 & & 0.0278 \\
\hline
\end{tabular}

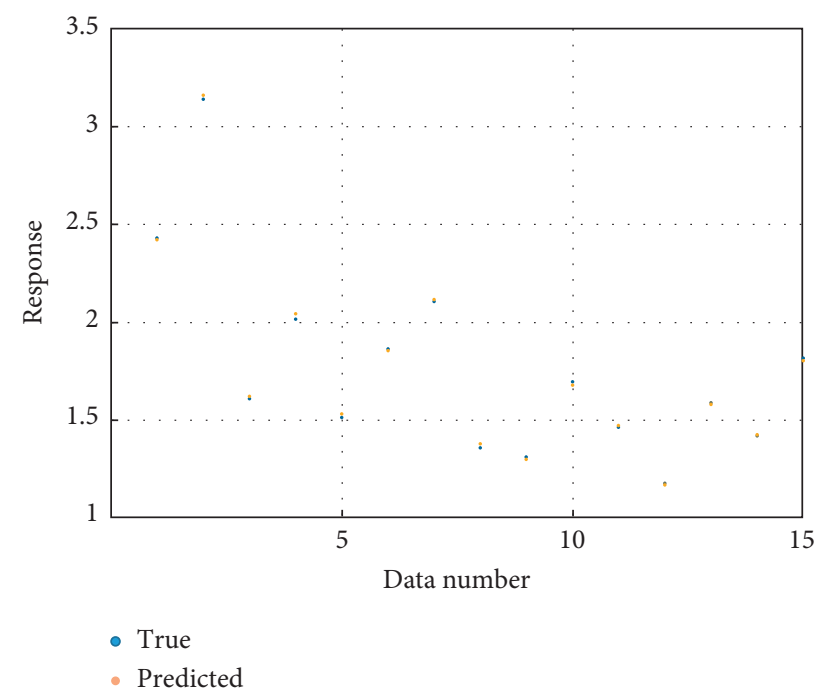

FIGURE 5: Scatter diagrams of simulated and computed resonant frequency values for test data (subsample 6).

compared with the literature. Comparative simulation and computed resonant frequency values are given in Table 4 . In this table, PQGR, fine TR, squared exponential GR, exponential GR, and KNN [26] results are given. Table 4 includes percentage errors for each test data.
Table 5 shows average percentage errors (APEs) of the four best-proposed algorithms. The APE values of PQGR, fine TR, squared exponential GR, and exponential GR are, respectively, 0.5981, 4.1606, 5.3533, and 7.3456. The obtained APE by PQGR is well below the obtained APE by KNN [26] 
TABLE 4: Comparative simulation and computed results.

\begin{tabular}{|c|c|c|c|c|c|c|c|c|c|c|c|c|c|c|}
\hline \multirow{2}{*}{ Sample number } & \multicolumn{7}{|c|}{ Patch dimension $(\mathrm{mm})$} & \multirow{2}{*}{$\begin{array}{l}\text { Sim. } \\
f_{r S I M}\end{array}$} & \multirow{2}{*}{$\begin{array}{l}\text { Mea. } \\
f_{r M E A}\end{array}$} & \multirow{2}{*}{ PQGR } & \multirow{2}{*}{ Fine TR } & \multirow{2}{*}{ Squared exponential GR } & \multirow{2}{*}{ Exponential GR } & \multirow{2}{*}{$\mathrm{KNN}[26]$} \\
\hline & $L$ & $W$ & $l$ & $w$ & $d$ & $h$ & $\varepsilon_{r}$ & & & & & & & \\
\hline 1 & 30 & 20 & 10 & 5 & 3 & 2.5 & 4.4 & 2.426 & - & 2.4202 & 2.3821 & 2.4588 & 2.3785 & 2.4100 \\
\hline 2 & 30 & 20 & 10 & 10 & 6 & 2.5 & 2.33 & 3.140 & - & 3.1575 & 3.1785 & 3.1402 & 2.9439 & 3.1500 \\
\hline 3 & 30 & 20 & 20 & 5 & 3 & 1.6 & 4.4 & 1.611 & - & 1.6200 & 1.5579 & 1.5293 & 1.7040 & 1.6300 \\
\hline 4 & 30 & 20 & 20 & 5 & 6 & 1.6 & 2.33 & 2.015 & - & 2.0416 & 2.0584 & 2.0345 & 2.1477 & 2.0500 \\
\hline 5 & 30 & 20 & 20 & 10 & 6 & 1.6 & 4.4 & 1.512 & - & 1.5276 & 1.5579 & 1.5384 & 1.6463 & 1.5400 \\
\hline 6 & 40 & 30 & 15 & 7.5 & 5 & 1.6 & 4.4 & 1.860 & - & 1.8539 & 1.6208 & 1.8302 & 1.8793 & 1.8700 \\
\hline 7 & 40 & 30 & 15 & 15 & 5 & 2.5 & 2.33 & 2.107 & - & 2.1134 & 2.2321 & 2.1814 & 2.0865 & 2.1000 \\
\hline 8 & 40 & 30 & 30 & 7.5 & 5 & 1.6 & 2.33 & 1.357 & - & 1.3736 & 1.3201 & 1.4843 & 1.5863 & 1.3800 \\
\hline 9 & 40 & 30 & 30 & 7.5 & 10 & 2.5 & 2.33 & 1.311 & - & 1.2962 & 1.3201 & 1.3085 & 1.5443 & 1.2800 \\
\hline 10 & 40 & 30 & 30 & 15 & 5 & 2.5 & 4.4 & 1.695 & - & 1.6787 & 1.7317 & 1.6202 & 1.4781 & 1.6700 \\
\hline 11 & 50 & 40 & 20 & 10 & 7 & 1.6 & 4.4 & 1.466 & - & 1.4699 & 1.2287 & 1.2366 & 1.4202 & 1.4900 \\
\hline 12 & 50 & 40 & 20 & 10 & 14 & 2.5 & 4.4 & 1.174 & - & 1.1689 & 1.2287 & 1.2406 & 1.3332 & 1.1600 \\
\hline 13 & 50 & 40 & 20 & 20 & 14 & 1.6 & 2.33 & 1.585 & - & 1.5821 & 1.5906 & 1.7255 & 1.6204 & 1.6000 \\
\hline 14 & 50 & 40 & 40 & 10 & 7 & 2.5 & 4.4 & 1.421 & - & 1.4230 & 1.3670 & 1.6788 & 1.5644 & 1.4100 \\
\hline 15 & 50 & 40 & 40 & 20 & 7 & 2.5 & 2.33 & 1.814 & - & 1.7984 & 1.8845 & 1.7772 & 1.6470 & 1.7900 \\
\hline 16 & 30 & 20 & 10 & 5 & 3 & 3 & 4 & 2.426 & 2.430 & 2.429 & 2.3821 & 2.4654 & 2.4336 & 2.4090 \\
\hline
\end{tabular}

TABle 5: Percentage errors.

\begin{tabular}{|c|c|c|c|c|c|}
\hline \multirow{2}{*}{ Sample number } & \multicolumn{5}{|c|}{ Percentage errors (\%) } \\
\hline & PQGR & Fine TR & Squared exponential GR & Exponential GR & KNN [26] \\
\hline 1 & 0.24 & 1.81 & 1.35 & 1.96 & 0.65 \\
\hline 2 & 0.55 & 1.22 & 0.002 & 6.25 & 0.31 \\
\hline 3 & 0.54 & 3.31 & 5.09 & 5.76 & 1.17 \\
\hline 4 & 1.31 & 2.14 & 0.95 & 6.57 & 1.73 \\
\hline 5 & 1.02 & 3.02 & 1.73 & 8.87 & 1.85 \\
\hline 6 & 0.32 & 12.85 & 1.59 & 1.05 & 0.53 \\
\hline 7 & 0.32 & 5.95 & 3.54 & 0.96 & 0.33 \\
\hline 8 & 1.24 & 2.70 & 9.41 & 16.92 & 1.69 \\
\hline 9 & 1.13 & 0.70 & 0.19 & 17.80 & 2.36 \\
\hline 10 & 0.97 & 2.16 & 4.42 & 12.80 & 1.47 \\
\hline 11 & 0.23 & 16.22 & 15.67 & 3.16 & 1.63 \\
\hline 12 & 0.41 & 4.68 & 5.70 & 13.59 & 1.19 \\
\hline 13 & 0.21 & 0.33 & 8.84 & 2.21 & 0.94 \\
\hline 14 & 0.16 & 3.78 & 18.17 & 10.11 & 0.77 \\
\hline 15 & 0.86 & 3.89 & 2.03 & 9.21 & 1.32 \\
\hline 16 & 0.06 & 1.81 & 1.62 & 0.31 & 0.70 \\
\hline Average & 0.5981 & 4.1606 & 5.3533 & 7.3456 & 1.1702 \\
\hline
\end{tabular}

in the literature. Therefore, it is seen that the PQGR method is more successful than other methods in calculating the resonant frequency when considering the 6-fold cross-validation results and the results of MAE, ME, MSE, RMSE, and APE in the subsample 6 test.

\section{Conclusion}

In this study, the resonant frequency for the C-like microstrip patch antenna was tried to be computed in a comparative analysis. Microstrip patch antennas were analyzed by the CEM simulation program, and the dataset was created. There are a total of 160 simulation data with different geometric and electrical properties. The PQGR model was trained with \# 145 training data and tested with subsample 6. The regression function for PQGR analysis was pure quadratic. It can analyze challenging datasets accurately. A single measurement data was also used in the test process. MAE, ME, MSE, RMSE, and APE were used to determine the performance of the PQGR model. The resonant frequency for subsample 6 was computed with 0.0109 MAE, 0.0087 ME, 0.0002 MSE, 0.0156 RMSE, and 0.5981 APE. Consequently, the obtained results in PQGR for both training and test data are very close to the simulation results compared to other regression models. Therefore, the PQGR method, which is proposed as an alternative to the highly costly simulation and measurement procedures, can calculate the resonant frequency with high accuracy.

\section{Data Availability}

The data used to support the findings of this study are available from the corresponding author upon request.

\section{Conflicts of Interest}

The authors declare that they have no conflicts of interest. 


\section{References}

[1] G. Kumar and K. P. Ray, Broadband Microstrip Antennas, Artech House, Massachusetts, MA, USA, 2003.

[2] K.-L. Wong, Compact and Broadband Microstrip Antennas, John Wiley and Sons, New York, NY, USA, 2002.

[3] G. Dubost and A. Rabbaa, "Analysis of a slot microstrip antenna," IEEE Transactions on Antennas and Propagation, vol. 34, no. 2, pp. 155-163, 1986.

[4] P. Shan-Cheng and W. Kin-Lu, "Dual-frequency triangular microstrip antenna with a shorting pin," IEEE Trans Antennas Propag, vol. 45, pp. 1889-1891, 1997.

[5] A. A. Deshmukh and G. Kumar, "Formulation of resonant frequency for compact rectangular microstrip antennas," Microwave and Optical Technology Letters, vol. 49, no. 2, pp. 498-501, 2007.

[6] Z. N. Chen, "Radiation pattern of a probe-fed L-shaped plate antenna," Microwave and Optical Technology Letters, vol. 27, no. 6 , pp. 410-413, 2000.

[7] A. Akdagli, A. Kayabasi, and I. Develi, "Computing resonant frequency of C-shaped compact microstrip antennas by using ANFIS," International Journal of Electronics, vol. 102, no. 3, pp. 407-417, 2015.

[8] N. Ojaroudi, M. Ojaroudi, and N. Ghadimi, "UWB omnidirectional square monopole antenna for use in circular cylindrical microwave imaging systems," IEEE Antennas and Wireless Propagation Letters, vol. 11, pp. 1350-1353, 2012.

[9] L. Liu, S. W. Cheung, R. Azim, and M. T. Islam, "A compact circular-ring antenna for ultra-wideband applications," $M i$ crowave and Optical Technology Letters, vol. 53, no. 10, pp. 2283-2288, 2011.

[10] J.-J. Tiang, M. T. Islam, N. Misran, and M. Singh, "Circular microstrip slot antenna for dual-frequency RFID application," Progress In Electromagnetics Research, vol. 120, pp. 499-512, 2011.

[11] Y. Ge, K. P. Esselle, and T. S. Bird, "E-shaped patch antennas for high-speed wireless networks," IEEE Transactions on Antennas and Propagation, vol. 52, no. 12, pp. 3213-3219, 2004.

[12] D. K. Neog, S. S. Pattnaik, D. C. Panda, S. Devi, M. Dutta, and O. P. Bajpai, "New expression for the resonant frequency of an E-shaped microstrip patch antenna," Microwave and Optical Technology Letters, vol. 48, no. 8, pp. 1561-1563, 2006.

[13] B. L. Ooi and Q. Shen, "A novel E-shaped broadband microstrip patch antenna," Microwave and Optical Technology Letters, vol. 27, no. 5, pp. 348-352, 2000.

[14] A. K. Bhattacharyya, R. Garg, and R. Garg, "Generalised transmission line model for microstrip patches," IEE Proceedings H Microwaves, Antennas and Propagation, vol. 132, no. 2, pp. 93-98, 1985.

[15] A. Taflove, Computational Electrodynamics: The Finite-Difference Time Domain Method, Artech House, Boston,USA, 1995.

[16] R. F. Harrington, Field Computation by Moment Methods, IEEE Press, Piscataway, NJ, USA, 1993.

[17] M. Paulson, S. O. Kundukulam, C. K. Aanandan, and P. Mohanan, "Resonance frequencies of compact microstrip antenna," Electronics Letters, vol. 37, no. 19, pp. 1151-1153, 2001.

[18] D. K. Neog and R. Devi, "Determination of resonant frequency of slot-loaded rectangular microstrip patch antennas," Microwave and Optical Technology Letters, vol. 52, pp. 446448, 2010.
[19] P. Palanisamy, I. Rajendran, and S. Shanmugasundaram, "Prediction of tool wear using regression and ANN models in end-milling operation," The International Journal of Advanced Manufacturing Technology, vol. 37, no. 1-2, pp. 29-41, 2008.

[20] S. Wang, L. Zhu, J. Y. H. Fuh, H. Zhang, and W. Yan, "Multiphysics modeling and Gaussian process regression analysis of cladding track geometry for direct energy deposition," Optics and Lasers in Engineering, vol. 127, Article ID 105950, 2020.

[21] S. Tong, X. Zhang, Z. Tong, Y. Wu, N. Tang, and W. Zhong, "Online ash fouling prediction for boiler heating surfaces based on wavelet analysis and support vector regression," Energies, vol. 13, no. 1, p. 59, 2020.

[22] A. Torres-Barrán, Á. Alonso, and J. R. Dorronsoro, "Regression tree ensembles for wind energy and solar radiation prediction," Neurocomputing, vol. 326-327, pp. 151-160, 2019.

[23] S. Ülker, "Support vector regression analysis for the design of feed in a rectangular patch antenna," in Proceedings of the $3 \mathrm{rd}$ International Symposium on Multidisciplinary Studies and Innovative Technologies (ISMSIT), IEEE, pp. 1-3, Ankara, Turkey, October 2019.

[24] K. V. Rop, Parameter Optimization in Design of a Microstrip Patch Antenna Using Adaptive Neuro-Fuzzy Inference System Technique, PhD Thesis, University of Nairobi, Kenya, 2014.

[25] F. Güneş, N. T. Tokan, and F. Gürgen, "A consensual modeling of the expert systems applied to microwave devices," International Journal of RF and Microwave ComputerAided Engineering, vol. 20, pp. 430-440, 2010.

[26] E. Yigit, "Operating frequency estimation of slot antenna by using adapted kNN algorithm," International Journal of Intelligent Systems and Applications in Engineering, vol. 6, no. 1, pp. 29-32, 2018.

[27] D. B. Davidson, Computational Electromagnetics for $R F$ and Microwave Engineering, Cambridge University Press, Cambridge, UK, 2005.

[28] E. M. HyperLynx ${ }^{\circledR}$ 3D, Version 15, Mentor Graphics Corporation, 8005 SW Boeckman Road, Wilsonville, OR 97070, USA.

[29] D. J. Cannon, D. J. Brayshaw, J. Methven, P. J. Coker, and D. Lenaghan, "Using reanalysis data to quantify extreme wind power generation statistics: a 33 year case study in Great Britain," Renewable Energy, vol. 75, pp. 767-778, 2015.

[30] C. Rasmussen and C. Williams, Gaussian Processes for $\mathrm{Ma}$ chine Learning, MIT Press, Massachusetts, MA, USA, 2006.

[31] A. J. Smola and B. Schölkopf, A Tutorial on Support Vector Regression, Technical Report NC-TR-98-030, NeuroCOLT Royal Holloway College, University of London, UK, 1998.

[32] Y. Chen, J. Wang, R. Xia, Q. Zhang, Z. Cao, and K. Yang, "The visual object tracking algorithm research based on adaptive combination kernel," Journal of Ambient Intelligence and Humanized Computing, vol. 10, no. 12, pp. 4855-4867, 2019.

[33] Y. Peng, K. Lei, X. Yang, and J. Peng, "Improved chaotic quantum-behaved particle swarm optimization algorithm for fuzzy neural Network and its application," Mathematical Problems in Engineering, vol. 2020, Article ID 9464593, 11 pages, 2020. 